\title{
A comparative study to evaluate the efficacy of ultrasound guided brachial plexus block and peripheral nerve block for upper limb surgeries
}

\author{
Mohammed Mukarram Iqbal ${ }^{1}$, Vikas S Joshi ${ }^{*}$, Kaja Sriram Murthy ${ }^{3}$ \\ 1,2 Assistant Professor, ${ }^{3}$ Professor and Head, Dept. of Anaesthesia, MRMC, Kalaburgi, Karnataka, India
}

*Corresponding Author: Vikas S Joshi

Email: vikassjoshi@gmail.com

Received: $2^{\text {nd }}$ February, 2019

Accepted: $12^{\text {th }}$ February, 2019

\begin{abstract}
Introduction: Ultrasound is gaining popularity in routine anesthetic practice both in operating room as well as in intensive care units. Brachial plexus block and peripheral nerve block in various combinations have been used successfully for upper limb surgeries. But the disadvantage of brachial plexus block is the inability to use the affected limb in the post operative period due to motor block. The present study was undertaken to assess the degree of motor sparring under USG guided peripheral nerve block as compared to USG guided brachial plexus block.

Materials and Methods: The study was a randomized open label study conducted in two groups viz. group A and group B. Patients in group A received treatment A i.e peripheral nerve block (PNB) and patients in group B received treatment B i.e brachial plexus block.

Results: In peripheral nerve block group the median strength loss was $23 \%$ whereas the same was $100 \%$ in brachial plexus block group. This difference was found to be statistically significant $(P=0.001)$. The anesthetic onset time was found to be significantly shorter in peripheral nerve block group as compared to brachial plexus group $(7.71 \pm 1.3 \mathrm{Vs} 9.58 \pm 1.91 \mathrm{~min})$. Subject's satisfaction score was reported higher with peripheral nerve block than those who underwent brachial plexus block: 5 Vs 4 respectively $(p=0.012)$. Likewise these satisfaction scores were found to have inverse correlation with loss of strength in the operative limb (Spearman's rho $-0.62[\mathrm{p}=0.016]$ and Kendall's tau $-0.55[\mathrm{p}=0.025])$.

Conclusion: Therefore we conclude that ultrasound guided peripheral nerve block can be an effective alternative to brachial plexus block as a primary mode of anesthesia in hand surgeries of short duration.
\end{abstract}

Keywords: Hand surgery, Peripheral nerve block, Brachial plexus block, Ultrasonography.

\section{Introduction}

Ultrasound (US) is gaining popularity in routine anesthetic practice both in operating room as well as in intensive care units. Over a period of time ultrasound has evolved to be an asset in the armamentarium of modern anesthesiologist. Ultrasound guided regional blocks are commonly performed for hand surgeries. A very important disadvantage of brachial plexus block is the loss of motor control in the operative hand which could result in less patient satisfaction. ${ }^{1,2}$ To address this issue some studies have been undertaken in past where peripheral nerve blocks have been used and they have shown promising results in improving the motor function. ${ }^{3,4}$ The present study was undertaken to assess the degree of motor sparring under ultrasonography (USG) guided peripheral nerve block (PNB) as compared to USG guided brachial plexus block.

\section{Materials and Methods}

Approval from the institutional ethics committee was obtained before the commencement of the study. The study was conducted at Basaveshwar Teaching and General Hospital attached to Mahadevappa Rampure Medical College, Kalaburagi, Karnataka. The patients who were included in the study were those aged 18-65 years, including both male and female gender, belonging to ASA (American Society of Anesthesiologist) I to III category. The patients were also selected based on the laterality i.e patients undergoing unilateral hand surgery requiring a procedure of less than $15 \mathrm{~min}$ duration. Written informed consent was obtained from all the participants who agreed to take part in the study. All those patients were excluded from study in whom surgery was planned outside the median and radial nerve distribution, patients who required surgery in both the hands, anatomical deformities of neck or clavicle, infection at the planned injection site, uncontrolled diabetes mellitus with complications, previous history of allergy to local anesthetic agents, any coagulopathy and patients who refused to sign the informed consent form. The study was a randomized open label study conducted in two groups viz. group A and group B. Patients in group A received treatment $\mathrm{A}$ i.e distal block (peripheral nerve block) and patients in group B received treatment B i.e proximal block (brachial plexus block). Randomization for group allocation was performed using a computer generated random number table to produce a series of numbers that were divided in two halves. The numbers falling in first half received treatment $\mathrm{A}$ while the numbers in second half received treatment B. In the pre-operative period all subjects were given etoricoxib $60 \mathrm{mg}$ orally unless contraindicated. A single anesthesiologist who was well versed with all kinds of regional anesthesia techniques performed both the blocks under ultrasound guidance preoperatively in a holding room. The motor function of study participants was assessed by testing their hand grip strength with hand dynamometer (JAMAR()). Initially baseline measurements were taken before surgery in both the hands to compare it with postoperative hand strength. Basic preoperative treatment like IV line, oxygen inhalation, pulse, Blood pressure, 
temperature monitoring, oxygen saturation etc was given to all the patients. IV Midazolam $2 \mathrm{mg}$ and fentanyl $25 \mathrm{mcg}$ was given to the patients for sedation and anxiolysis.

Peripheral nerve block procedure: The patients who were included in Group A were placed in the supine position and the arm that was to be blocked was abducted and rotated externally. The nerves were visualised under US before performing nerve blocks and the skin was marked where the radial and median nerves could be viewed clearly at the mid-forearm or above (Fig. 1). ${ }^{5,6}$ After disinfection of the puncture site using chlorhexidine, a US probe (Sonosite, WA) was dressed with a sterile cover and placed at the marked level of the forearm. At first, a subcutaneous local anesthetic was injected. Secondly, with a $22 \mathrm{G} 50 \mathrm{~mm}$ USvisible needle (SonoPlex Stim Cannula; Germany), $5 \mathrm{~mL}$ local anesthetic (mixture of $0.5 \%$ bupivacaine and $2 \%$ lidocaine) was injected to block each nerve (total $10 \mathrm{ml}$ ). The radial nerve was located lateral to the radial artery. The US probe was placed on the marked area of skin of the forearm, the needle was inserted and advanced from the lateral to the medial side towards the radial nerve using an in-plane technique, and $5 \mathrm{~mL}$ local anesthetic was injected around the radial nerve. The median nerve block was performed without removing the needle at the same puncture site. When the US probe was moved from the lateral to the medial side, the median nerve was readily viewed, the needle was advanced toward the median nerve, and $5 \mathrm{~mL}$ local anesthetic was injected. The needle was redirected to view the proper spread of the local anesthetic (Fig. 2).

\section{Brachial Plexus Block Procedure}

The patients who were included in Group B were placed in the supine position and the head of the patient was turned towards the side opposite to the operation site. The brachial plexus was visualised under US around the axillary artery before performing brachial plexus block. ${ }^{7,8}$ After disinfection of the puncture site using chlorhexidine, a linear US probe was dressed with a sterile cover and placed in a sagittal plane. Following subcutaneous infiltration of local anesthetic, a 22G $80 \mathrm{~mm}$ US-visible block needle (SonoPlex Stim Cannula; Germany) was placed between the posterior cord and the axillary artery, $20 \mathrm{~mL}$ local anesthetic (mixture of $0.5 \%$ bupivacaine and $2 \%$ lidocaine) was administered, and the U-shaped spread of the local anesthetic around the axillary artery was viewed under US guidance.

The block procedure time defined as the time interval between the first contact of US transducer to the time when block needle was taken out of the skin was recorded. After completion of procedure the sensory blockade was evaluated every 5 minutes with ice cube on the palmar surface of index finger and little finger; whereas motor block was assessed only before the block and in the postoperative period. The subjects in whom complete sensory blockade was not achieved within 30 minutes were categorised as block failure and excluded from the study. A pneumatic tourniquet was applied on the operative limb and patients were sedated with IV midazolam. Short-acting opioids were given to the patients who complained of pain during surgery. No other anesthetics or analgesics were allowed to be used during the surgery. Patients in whom more than normal sedation or any other supplementary block was required were noted down. In the post operative ward the time when patients first felt their hand numbness resolving was noted down adequate analgesia was provided to them for any breakthrough pain occurring thereafter. Simultaneously the motor function was also assessed using the same hand held dynamometer as in the preoperative period and the hand grip strength was recorded.

The primary aim of our study was to assess the change in hand grip strength of operative limb as compared to preoperative baseline strength. There were many secondary outcome measures such as block procedure time, success or failure of block, time to anesthetic onset, breakthrough sedatives and analgesics required if any during intra or post operative period, surgeon's satisfaction with respect to operative conditions with both the blocks and patient's satisfaction with respect to their ability to move the operative hand and their overall experience with the respective block procedures received. To assess the surgeon's and patient's satisfaction a five point Likert's scale was used as explained here; A scale of 1 to 5 was used with 1 being not at all satisfied, 3 being neutral, and 5 being completely satisfied, the question for surgeons read as, "How satisfied were you with the surgical conditions provided for this subject?" and the question of patients read as, "Thinking about your nerve blocks, how satisfied were you with them". Patients were also asked about the time when they first felt their numbness resolving and when did they first felt the full recovery of the sensation in the operated hand. The participants were asked to report any adverse reactions experienced related to their anesthetic procedure such as paraesthesia, motor deficits, persistant pain, bruising at the site of injection etc during the post operative period.

\section{Statistical Analysis}

Assuming a difference in the effect size of previous studies and to give $80 \%$ power, and $\alpha$ error of $0.05,26$ subjects with additional 10-15\% scope for loss to follow-up, a sample size of 30 (15 in each group) was estimated to give the desired results. Continuous data with normal distribution were presented as mean (standard deviation [SD]) and analyzed with Student's $t$-test; continuous data with non normal distribution were presented as median (interquartile range $[\mathrm{IQR}]$ ) and analyzed using the Mann-Whitney $U$ test. Categorical data were analyzed with the Chi-square test. Correlation between change in hand grip strength and patient satisfaction was assessed with two nonparametric measures of association: Spearman's rho and Kendall's tau. A $P$ value of $<0.05$ was considered statistically significant. 


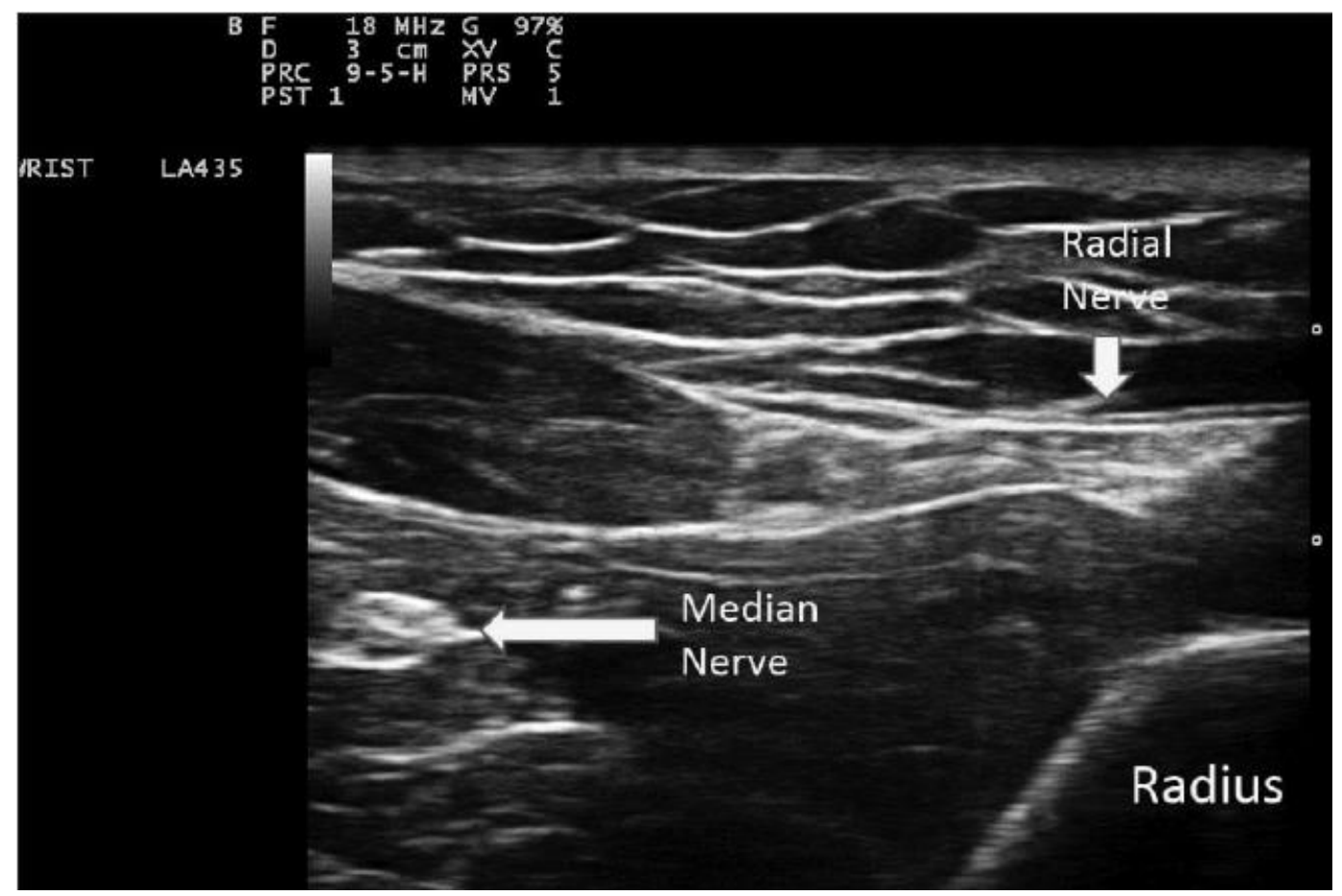

Fig. 1: Simultaneous visualization of the radial and median nerves on an ultrasound screen

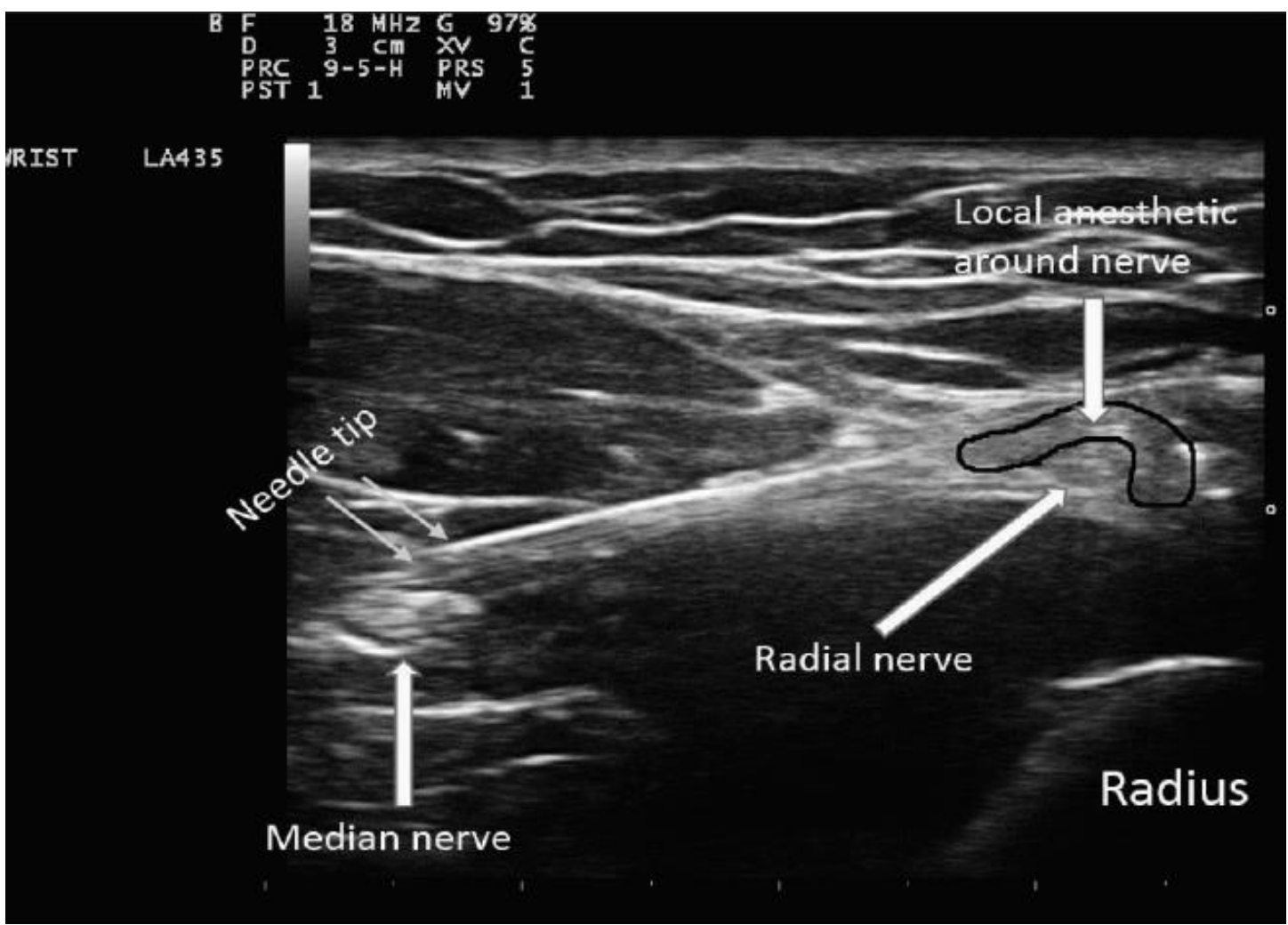

Fig. 2: Position of radial nerve, median nerve, and needle

\section{Results}

Out of the 41 patients who were assessed for their eligibility, 3 of them refused to participate whereas 8 patients were excluded after failed to meet the inclusion criteria. Therefore remaining 30 patients were enrolled into the study and all these subjects successfully completed the study (Fig. 3). 


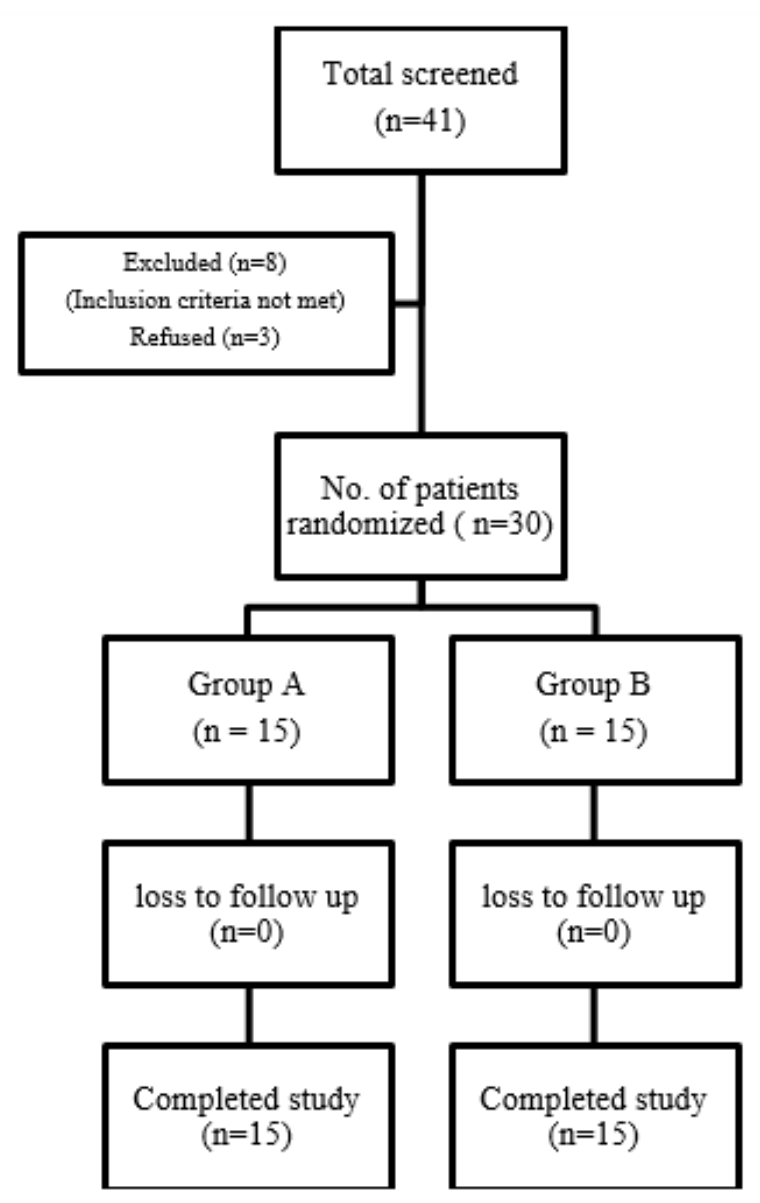

Fig. 3: Randomization chart

Table 1 shows demographic data of patients in both the study groups. Their characteristics did not have any difference of statistical significance. None of the patients in both the groups reported block failure and there were no reports of any breakthrough analgesia or additional anesthetics being used in any patient intraoperatively and in post operative period.

Table 1: Demographic data and surgical observations

\begin{tabular}{|l|c|c|}
\hline & $\begin{array}{c}\text { Distal group }(\boldsymbol{n} \\
\mathbf{= 1 5})\end{array}$ & $\begin{array}{c}\text { Proximal group }(\boldsymbol{n} \\
\mathbf{1 1 5})\end{array}$ \\
\hline Age, years & $54.0(5.1)$ & $53.7(5.6)$ \\
\hline Sex, $n(\mathrm{~m} / \mathrm{f})$ & $7 / 8$ & $8 / 7$ \\
\hline Height, $\mathrm{cm}$ & $163.0(9.1)$ & $164.7(11.8)$ \\
\hline Weight, kg & $86.5(16.2)$ & $87.6(17.1)$ \\
\hline
\end{tabular}

\section{Primary Outcome}

In group A I.e the distal block group, median strength loss was $23 \%(14.5 \%, 48.1 \%)$ in operative hand in the post operative period as compared to preoperative strength; whereas in Group B I.e brachial plexus block group the strength loss was $100 \%(100 \%, 100 \%)$. This difference between both the groups was found to be statistically significant with a $\mathrm{p}$ value of 0.001 .

\section{Secondary Outcomes}

The time taken for block procedure was shorter for brachial plexus as compared to peripheral nerves block $(106.53 \pm 31.8$ seconds Vs $112.6 \pm 33.1$ seconds respectively). However this difference in time was not statistically significant. The anesthetic onset time was significantly shorter in peripheral nerve block as compared to brachial plexus block $(7.71 \pm 1.3$ minutes Vs. $9.58 \pm 1.91$ minutes respectively, $\mathrm{p}=0.002$ ).

A higher satisfaction score was reported by participants in PNB group as compared to brachial plexus block group $(5[5,5]$ Vs. $4[4,4]$ respectively). The difference in this score was found to be statistically significant with $\mathrm{p}$ value of 0.012. These subject satisfaction scores were found to inversely correlated to loss of strength in the operative hand (Spearman's rho-0.62[p=0.01] and Kendall's tau $0.55[\mathrm{p}=0.02])$. The mean time for return of sensation in group A was 261(91.6) minutes while in group B it was 358 (53.5) minutes $(\mathrm{p}=0.03)$. No significant difference was found in other secondary outcomes (table 2). Bruising was seen in 3 and 2 patients from distal and peripheral block respectively. Two subject complained of short lasting paraesthesia in the distal group, while in proximal block group one person had paraesthesia which resolved by the second postoperative day.

\section{Discussion}

Minor hand surgeries that require short time can be done under brachial plexus block as well as peripheral nerve blocks both with the aid of ultrasound guidance. But the disadvantage of the former technique is loss of motor function in the operated limb post surgery ultimately reducing the patient satisfaction with the treatment received. The present study is one of the few studies done to directly compare the efficacy of brachial plexus block and peripheral nerve block as a primary method of anesthesia both under ultrasound guidance for short lasting unilateral hand surgeries. In our study it was observed that distal peripheral nerve blocks are superior at preserving motor function of the operative limb which reflected well in patient satisfaction scores where modest improvements were seen. The present study was designed based on the assumption that preservation of motor function of hand during and after surgery would lead to better patient satisfaction, the feasibility of which is supported by various published material for minor hand surgery. ${ }^{9-11}$ Several studies in the past have shown that local infiltration of anesthetic and IV regional anesthesia (Bier's block) can also be done for minor hand surgeries. ${ }^{12,13}$

One of the main advantages of peripheral nerve block is the preservation of motor function for intra operative assessment of hand movement when instructed to do so. It preserves the protective reflexes and the need for a sling in the post operative period and also the cost associated with it thus avoiding the unanticipated injury to the operative limb. ${ }^{14}$ 
Table 2: Secondary outcome observations

\begin{tabular}{|l|c|c|c|}
\hline & $\begin{array}{c}\text { Group A }(\boldsymbol{n}=\mathbf{1 5}) \\
\text { (Distal block) }\end{array}$ & $\begin{array}{c}\text { Group B }(\boldsymbol{n}=\mathbf{1 5}) \\
\text { (proximal block) }\end{array}$ & $\boldsymbol{P}$ value \\
\hline Anesthetic onset time, min & $7.71+1.3$ & $9.58+1.91$ & 0.002 \\
\hline Block procedure time (sec) & $112.6+33.1$ & $106.53+31.8$ & 0.31 \\
\hline Surgery duration (min) & $12.1(2.6)$ & $15.7(4.5)$ & 0.09 \\
\hline Tourniquet duration, min & $8.0(2.2)$ & $10.0(3.4)$ & 0.21 \\
\hline Surgeon's satisfaction, $1-5$ & $5(5,5)$ & $5(5,5)$ & 0.39 \\
\hline Subject's satisfaction, $1-5$ & $5(5,5)$ & $4(4,4)$ & 0.01 \\
\hline Return of sensation (min) & $261(91.6)$ & $358(53.5)$ & 0.03 \\
\hline Bruising, $n$ & 3 & 2 & 0.99 \\
\hline Paresthesia, $n$ & 2 & 1 & 0.99 \\
\hline
\end{tabular}

$\mathrm{n}=$ Number of subjects

The reason for including the patients whose surgery was planned in the median and radial nerve distribution was to perform only one puncture in Group A. The block selection was restricted to radial and medial nerve in order to achieve standardization in the study. In our opinion, if the number of punctures increases there were chances that the number of complications will increase and patient satisfaction could decrease eventually.

In our study though the block procedure time for brachial plexus block was shorter the difference was found not to be statistically significant. However, the time for anesthetic onset was significantly shorter in distal group than in proximal block group. In this study, we administered $20 \mathrm{~mL}$ of a local anesthetic mixture (1:1 lidocaine/ bupivacaine) for infraclavicular block, $5 \mathrm{~mL}$ of $1: 1$ lidocaine/bupivacaine for radial nerve block, and $5 \mathrm{~mL}$ of 1:1 lidocaine/bupivacaine for median nerve block. The total amount of local anesthetic was $10 \mathrm{~mL}$ in Group A. In the study performed by Soberon et al. the average volume of local anesthetic was $15.5 \mathrm{~mL}$ in the forearm block group and $29.9 \mathrm{~mL}$ in the supraclavicular block group, with various combinations of local anesthetic. ${ }^{15}$ In another study conducted by Lam et al., the volume of local anesthetic was $10 \mathrm{~mL}$ for selective nerve block $(5 \mathrm{~mL}$ for the ulnar and 5 $\mathrm{mL}$ for the median nerve) and $15 \mathrm{~mL}$ for supraclavicular block. ${ }^{16}$ The avoidance of local anesthetic toxicity after nerve block is an important benefit, and the volume of local anesthetic in the proximal block group is greater than that in the distal peripheral nerve block group, according to these studies. Since the volume of local anesthetic injected was larger in the brachial plexus block as compared to peripheral nerve block, the risk of local anesthetic toxicity was also higher with the former technique. None of the patients in either group had block failure or required rescue analgesics in post operative ward, therefore the observed difference in patient satisfaction core was unlikely to be related to difference in the anestheic technique used. In contrast to our findings Frederickson et al. did not find a statistically significant difference in patient satisfaction when they compared the two anesthetic techniques. ${ }^{17}$ According to the method of the current study, we examined the nerves with US before performing the nerve blocks. Our performance time for nerve block was similar in both groups.
Identification of nerves by US before the nerve block procedure shortens the performance time. In our study we did not observe any major anesthetic complications in group A or group B apart from the minor ones as listed in table 2 . Some important complications of brachial plexus block are pneumothorax, arterial puncture, hematoma, and Horner's syndrome. ${ }^{18}$ There is no risk of major complications such as pneumothorax and Horner's syndrome with distal peripheral nerve block. This feature of peripheral nerve block will definitely serve as an important advantage during the surgery.

\section{Conclusion}

Therefore we conclude that ultrasound guided peripheral nerve block can be an effective alternative to brachial plexus block as a primary mode of anesthesia in hand surgeries of short duration, as it avoids most of its disadvantages and also preserves the motor function to a greater extent ultimately leading to better patient satisfaction.

\section{Study Limitations}

The study was an open label study as the logistics and other ethical constraints did not support a blinded study. Another drawback of peripheral nerve block is that in surgical procedures that involve the innervation area of three or more nerves in the arm, forearm, or hand, multiple blocks can be uncomfortable to the patients because of increase in the number of punctures thereby reducing the patient satisfaction. Our primary outcome is assessed by hand grip strength, which may be subjective and patient's stamina is likely to influence their performance before and after surgery. So we took average of three recordings to assess the grip strength pre and postoperatively. The results of the present study may not be completely generalized as the technique may not be performed in exactly the same way as in present study and the operative and preoperative conditions may also be variable at different places.

Conflict of Interests: None. 


\section{References}

1. M. J. Fredrickson and D. J. Price. Analgesic effectiveness of ropivacaine $0.2 \%$ vs. $0.4 \%$ via an ultrasound-guided C5- 6 root/superior trunk perineural ambulatory catheter. $\mathrm{Br} \mathrm{J}$ Anaesth 2009;103(3):434-43.

2. M. J. Fredrickson, K. R. Smith, and A. C. Wong. Importance of volume and concentration for ropivacaine interscalene block in preventing recovery room pain and minimizing motor block after shoulder surgery. Anesthesiol 2009;112(6):1374-81.

3. E. R. Mariano, M. K. Lehr, V. J. Loland, and M. L. Bishop. Choice of loco-regional anesthetic technique affects operating room efficiency for carpal tunnel release. $J$ Anesth 2013;27:611-14.

4. P. Macaire, F. Singelyn, P. Narchi, and X. Paqueron. Ultrasound-or nerve stimulation-guided wrist blocks for carpal tunnel release: a randomized prospective comparative study. Reg Anesth Pain Med 2008;33(4)363-8.

5. C. J. L. McCartney, D. Xu, C. Constantinescu, S. Abbas, and V. W. S. Chan. Ultrasound examination of peripheral nerves in the forearm. Reg Anesth Pain Med 2007;32(5)434-9.

6. A. Kathirgamanathan, J. French, G. L. Foxall, J. G. Hardman, and N. M. Bedforth. Delineation of distal ulnar nerve anatomy using ultrasound in volunteers to identify an optimum approach for neural blockade. Eur J Anaesthesiol 2009;26(1):43-6.

7. L. G. Soares, R. Brull, J. Lai, and V. W. Chan. Eight ball, corner pocket: the optimal needle position for ultrasound guided supraclavicular block. Reg Anesth Pain Med 2007;32(1):94-5.

8. S. Kapral, P. Krafft, K. Eibenberger, R. Fitzgerald, M. Gosch, and C. Weinstabl. Ultrasound-guided supraclavicular approach for regional anesthesia of the brachial plexus. Anesth Analgesia 1994;78(3):507-13.

9. J. A. Dilger and R. E. Wells Jr. The use of peripheral nerve blocks at the elbow for carpal tunnel release. J Clin Anesth 2005; 17(8)621-3.

10. P. Macaire, O. Choquet, D. Jochum, V. Travers, and X. Capdevila. Nerve blocks at the wrist for carpal tunnel release revisited: The use of sensory-nerve and motor-nerve stimulation techniques. Reg Anesth Pain Med 2005;30(6):53640 .
11. L. Delaunay and J. E. Chelly. Blocks at the wrist provide effective anesthesia for carpal tunnel release. Can J Anesth 2001;48(7):656-60.

12. M. M. Tomaino, D. Ulizio, and M. T. Vogt. Carpal tunnel release under intravenous regional or local infiltration anaesthesia. J Hand Surg 2001;26(1)67-8.

13. S. Brill, W. Middleton, G. Brill, and A. Fisher. Bier's block; 100 years old and still going strong! Acta Anaesthesiologica Scand 2004;48(1)117-22.

14. A. Saporito, E. Sturini, J. Petri, A. Borgeat, and J. A. Aguirre. Case report: unusual complication during outpatient continuous regional popliteal analgesia. Can J Anesth 2012;59:958-62.

15. Soberón JR, Bhatt NR, Nossaman BD, Duncan SF, Patterson ME, Sisco-Wise LE. Distal peripheral nerve blockade for patients undergoing hand surgery: a pilot study. Hand $(N Y)$ 2015; 10:197-204.

16. Lam NC, Charles M, Mercer D. A triple-masked, randomized controlled trial comparing ultrasound-guided brachial plexus and distal peripheral nerve block anesthesia for outpatient hand surgery. Anesthesiol Res Pract 2014;324083:7.

17. Fredrickson MJ, Ting FS, Chinchanwala S, Boland MR. Concomitant infraclavicular plus distal median, radial, and ulnar nerve blockade accelerates upper extremity anaesthesia and improves block consistency compared with infraclavicular block alone. Br J Anaesth 2011;107:236-42.

18. Tobias JD. Brachial plexus anaesthesia in children. Paediatr Anaesth 2001;11:265-75.

How to cite this article: Iqbal MM, Joshi VS, Murthy KS. A comparative study to evaluate the efficacy of ultrasound guided brachial plexus block and peripheral nerve block for upper limb surgeries. Indian $J$ Clin Anaesth 2019;6(2):248-53. 